\title{
Neck Lymph Node Boundary Sublevel IIB
}

National Cancer Institute

\section{Source}

National Cancer Institute. Neck Lymph Node Boundary Sublevel IIB. NCI Thesaurus.

Code C132498.

The following anatomical structures define the neck lymph node boundary for sublevel IIB: Superior: skull base; inferior: horizontal plane defined by the inferior body of the hyoid bone; anterior (medial): vertical plane defined by the spinal accessory nerve; posterior (lateral): lateral border of the sternocleidomastoid muscle. (AJCC 8th ed.) 